ISSN 0258-7122

Bangladesh J. Agril. Res. 39(4): 675-683, December 2014

\title{
EVALUATION OF INBRED LINES OF MAIZE (Zea mays L.) THROUGH LINE $\times$ TESTER METHOD
}

\author{
M. N. AMIN ${ }^{1}$, M. AMIRUZZAMAN ${ }^{2}$, A. AHMED ${ }^{3}$ AND M. R. ALI ${ }^{4}$
}

\begin{abstract}
Maize inbred lines were evaluated by using line $\times$ tester method involving 11 lines and 3 testers for grain yield and its components through estimation of general combining ability (gca) and specific combining ability (sca) effects. Highly significant genotypic differences were observed indicated wide range of variability present among the genotypes. The crosses with high sca effect for grain yield were evolved from high $\times$ low general combiner parents which reveled additive $\times$ dominance type of gene action. The cross combinations 9MS4- $1 \times$ L22, 9MS4-1 × L486, 9MS4-2 × L431, 9MS4-11 × L486 and 9MS4$15 \times$ L431 with high positive sca effect having high mean values might be used for obtaining high yielding hybrids. The information on the nature of gene action with respective variety and characters might be used depending on the breeding objectives.
\end{abstract}

Keywords: Maize (Zea mays L.), Line $\times$ tester method, inbred lines, combining ability.

\section{Introduction}

Maize (Zea mays L.) is one of the important food and forage crops with abundant natural diversity. It is one of the most important cereal and ranks next to rice and wheat in production. Maize is a highly allogamous crop and it has been successfully exploited for the production of hybrids. Parental selection is very important in hybrid development. In this context, $\mathrm{L} \times \mathrm{T}$ analysis (Kempthome, 1957) has widely been used for evaluation of inbred lines by crossing them with testers. The value of any inbred line in hybrid breeding ultimately depends on its ability to combine very well with other lines to produce superior hybrids. Hence, combining ability is a useful biometric tool to the plant breeders for formulating an efficient breeding programme. Exploitation of hybrid vigor and selection of parents based on combining ability has been used as an important breeding approach in crop improvement. Line $\times$ tester analysis is an important method randomly used to evaluate the inbred lines. Through line $\times$ tester analysis about $50 \%$ of the inbred lines can be eliminated (Singh and Chaudhary, 1979). This

\footnotetext{
${ }^{1}$ Scientific officer, Plant Breeding Division, Bangladesh Agricultural Research Institute, Gazipur-1701, ${ }^{2}$ Principal scientific officer, Plant Breeding Division, Bangladesh Agricultural Research Institute,Joydebpur,Gazipur-1701, ${ }^{3}$ Scientific officer, Plant Breeding Division, Bangladesh Agricultural Research Institute Gazipur-1701, ${ }^{4}$ Chief scientific officer, Plant Breeding Division, Bangladesh Agricultural Research Institute, Gazipur-1701, Bangladeh.
} 
reduces the number of inbred lines to a manageable size for the next step. The present study involving a line $\times$ tester analysis aimed to determine the general combining ability (gca) of lines and specific combining ability (sca) of crosses for different traits and to explore heterotic hybrid combinations. It has been reported that selection during inbreeding based on the performance of test cross progeny is highly effective in improving the gca of inbred lines. gca of inbred lines can be effectively tested at an early stage during the inbreeding programme.

\section{Materials and method}

The experimental material comprised of eleven $\mathrm{S}_{5}$ generation lines (as female parents) and 3 testers (male parents) of maize with diverse genetic base. The 33 $(11 \times 3)$ cross combinations were recovered through Line $\times$ Tester mating design. The $33 \mathrm{~F}_{1}$ 's and 14 parental lines including three testers were grown in a randomized block design with two replications with spacing of $60 \times 20 \mathrm{~cm}$ at the Bangladesh Agricultural Research Institute, Joydebpur during rabi 2012. Two border rows were used at each end of the replication for minimize the border effect. All the recommended package of practices were followed and the observations were recorded on ten randomly selected plants for quantitative characters viz. days to $50 \%$ tasselling, days to $50 \%$ silking, plant height, ear length, kernel per ear, 1000 grain weight, and grain yield. Combining ability analysis was done as per the method given by Kempthrone (1957) and the results are presented in Tables 1-4.

\section{Results and Discussion}

The analysis of variance revealed significant differences among the genotypes for all the characters studied indicating sufficient genetic variability among the genotypes (Table-1). The analysis of variance for combining ability revealed significant differences in the variance due to lines, testers, hybrids and Line $\mathrm{x}$ Testers for all the traits (Table 1). Similar genotypic difference for ear length, grain weight, grain yield and other characters were reported by Sofi and Rathor (2006) and Narro et al. (2003). Highly significant differences present between parents due to all the traits. Significant differences were also observed between interactions of parent $\times$ hybrid for all traits, indicated wide range of variability present among them. Significant differences were also observed between the hybrids, indicated wide range of variability prevailed among them for the traits. Significant differences were observed between the lines for all the traits except yield indicated substantial variability prevailed in the lines. A significant difference was also existed between testers for days to silking, plant height, ear height, grains/ear and yield except days to tassel and 1000 grain weight. The interaction of line $\times$ tester also showed significant differences in 1000 grain weight and yield. The higher estimation of dominance variance as compared to additive variance for 1000 seed weight was probably due to predominance of 
non-additive gene action which suggests scope of improvement of these characters through heterosis breeding. Similar non-additive gene action was also reported by Suneetha et al. (2000) for days to 50\% tasselling and days to $50 \%$ silking. Singh and Singh (1998) reported non -additive gene action for plant height, ear length, kernel rows, 1000 grain weight and Mahto and Ganguly (2001) reported non additive gene action for grain yield.

Table 1. Mean squares and estimates of variance for grain yield, yield components and other characters in maize.

\begin{tabular}{|c|c|c|c|c|c|c|c|c|}
\hline Source & df & $\begin{array}{c}\text { Days to } \\
\text { tassel }\end{array}$ & \begin{tabular}{|c|}
$\begin{array}{c}\text { Days to } \\
\text { silk }\end{array}$ \\
\end{tabular} & $\begin{array}{c}\text { Plant } \\
\text { height }\end{array}$ & $\begin{array}{c}\text { Ear } \\
\text { height }\end{array}$ & Grains/ ear & \begin{tabular}{|c|}
$\begin{array}{c}\text { 1000- G. } \\
\text { Wt. }\end{array}$ \\
\end{tabular} & $\begin{array}{l}\text { Yield } \\
(\mathrm{t} / \mathrm{ha})\end{array}$ \\
\hline Genotypes & 46 & $29 * *$ & $24 * *$ & $1541 * *$ & $639 * *$ & $14810.3^{* *}$ & $1857 * *$ & $6.2 * *$ \\
\hline Parents (P) & 13 & $27 * *$ & $21 * *$ & $455^{*}$ & $238 * *$ & $11411.6^{*}$ & $1105 *$ & $5.9 * *$ \\
\hline $\mathrm{P}$ vs $\mathrm{C}$ & 1 & $608 * *$ & $567 * *$ & $51879 * *$ & $18175^{* *}$ & $349962.7 * *$ & $7693 * *$ & $30.3 * *$ \\
\hline Crosses(C) & 32 & $11 * *$ & $8.8 * *$ & $409 *$ & $253 * *$ & 5717.6 & $1980 * *$ & $5.6 * *$ \\
\hline Lines (L) & 10 & $22 * *$ & $16 * *$ & $866^{* *}$ & $569 * *$ & $7315.9 *$ & $2998^{*}$ & 7.7 \\
\hline Testers $(\mathrm{T})$ & 2 & 42 & $31 * *$ & $658 *$ & $376 * *$ & $21519.5^{* *}$ & 1135 & $4.8 * *$ \\
\hline $\mathrm{L} \times \mathrm{T}$ & 20 & 3.3 & 2.7 & 156 & 83 & 3338.2 & $1555^{*}$ & $4.6^{*}$ \\
\hline Error & 46 & 3.1 & 2.7 & 204 & 79 & 4620.0 & 726 & 2.2 \\
\hline \multicolumn{9}{|c|}{ Estimates of component of variances } \\
\hline$\sigma^{2} \mathrm{~g}$ (Line) & - & 3.2 & 2.3 & 118.4 & 80.9 & 663.0 & 240.5 & 0.5 \\
\hline$\sigma^{2} \mathrm{~g}($ Tester $)$ & - & 1.8 & 1.3 & 22.9 & 13.3 & 826.4 & -19.1 & 0.0 \\
\hline$\sigma^{2}$ gca & - & 0.2 & 0.2 & 6.5 & 4.4 & 61.0 & 10.9 & 0.0 \\
\hline$\sigma^{2}$ sca & - & 0.1 & 0.0 & -24.0 & 2.2 & -640.9 & 414.6 & 1.2 \\
\hline $\begin{array}{l}\sigma^{2} \text { gca/ } \\
\sigma^{2} \text { sca }\end{array}$ & - & 1.0 & - & -0.3 & 2.0 & -0.1 & 0.0 & 0.0 \\
\hline
\end{tabular}

$* \mathrm{P}=0.05$ and $* * \mathrm{P}=0.01$

The contribution of lines, testers and their interactions to total variances are presented in Table 2. The proportional contribution of lines and interactions to total variances, except days to tassel and silk, was much higher than testers in all the traits. However, the contribution of lines was higher than the tester to total variances for all the characters. These suggest female parent contributed maximum to total variance in maize, which was followed by interaction. Testers contributed lowest to total variance, which is in conformity with Parvin (2009).

Table 2. Proportional contribution of lines, testers and their interactions to total variance in maize.

\begin{tabular}{l|c|c|c|c|c|c|c|c}
\hline Source & $\begin{array}{c}\text { Days to } \\
\text { tassel }\end{array}$ & $\begin{array}{c}\text { Days to } \\
\text { silk }\end{array}$ & $\begin{array}{c}\text { Plant } \\
\text { height }\end{array}$ & $\begin{array}{c}\text { Ear } \\
\text { height }\end{array}$ & $\begin{array}{c}\text { Ear } \\
\text { length }\end{array}$ & $\begin{array}{c}\text { Grains/ } \\
\text { ear }\end{array}$ & $\begin{array}{c}\text { 1000- } \\
\text { GWt. }\end{array}$ & Yield \\
\hline $\begin{array}{l}\text { Due to line } \\
60\end{array}$ & 59 & 66 & 70 & 26 & 400 & 47 & 43 \\
$\begin{array}{l}\text { Due to tester } \\
\begin{array}{l}\text { Due to line } \\
\text { tester }\end{array}\end{array}$ & 23 & 22 & 10 & 9 & 16 & 235 & 4 & 5 \\
\hline
\end{tabular}




\section{General combining ability effects}

Selection of parents with good general combining ability is a prime requisite for any successful breeding programme especially for heterosis breeding. The general combining ability effects and per se performance of parents (line and tester) are presented in Table 3.

Table 3. General combining ability (gca) effects and mean of parents for grain yield, yield components and other characters in maize.

\begin{tabular}{|c|c|c|c|c|c|c|c|c|}
\hline \multirow[t]{2}{*}{ Parents } & \multicolumn{2}{|c|}{ Days to tasseling } & \multicolumn{2}{|c|}{ Days to silking } & \multicolumn{2}{|c|}{ Plant height } & \multicolumn{2}{|c|}{ Ear height } \\
\hline & gca & mean & gca & mean & gca & mean & gca & mean \\
\hline \multicolumn{9}{|l|}{ Testers: } \\
\hline 1.L 22 & 0.48 & 91 & 0.31 & 98.5 & 3.22 & 131 & 4.77 & 75 \\
\hline 2.CML 431 & 1.07 & 98 & 1.00 & 103.5 & -6.31 & 117 & -2.22 & 71 \\
\hline 3.CML 486 & -1.56 & 92 & -1.31 & 100 & 3.09 & 132 & -2.54 & 54.5 \\
\hline $\mathrm{SE}_{\left(g_{i}\right)}$ & 0.377 & - & 0.349 & - & 3.046 & - & 1.899 & - \\
\hline $\mathrm{SE}_{\left(g_{i-} g_{j}\right)}$ & 0.534 & - & 0.494 & - & 4.307 & - & 2.686 & - \\
\hline \multicolumn{9}{|l|}{ Lines: } \\
\hline 1. 9MS4-1 & $-1.65 * *$ & 101 & $-1.59 * *$ & 107 & 4.94 & 128.5 & $7.44 * *$ & 59.5 \\
\hline 2. $9 \mathrm{MS} 4-2$ & 0.35 & 103 & 0.24 & 109 & -1.89 & 114.5 & 0.61 & 50 \\
\hline 3. $9 \mathrm{MS} 4-4$ & $-2.32 * *$ & 98 & $-2.09 * *$ & 105 & $-10.06 * *$ & 116 & $-13.89 * *$ & 40.5 \\
\hline 4. $9 \mathrm{MS} 4-5$ & $-2.49 * *$ & 94 & $-2.09 * *$ & 101 & $-9.73 * *$ & 141 & $-4.73 * *$ & 66 \\
\hline 5. 9MS4-7 & 0.18 & 99 & -0.42 & 105 & $-20.89 * *$ & 111 & $-9.73 * *$ & 54.5 \\
\hline 6. 9MS4-9 & $1.68 * *$ & 98 & $1.58 * *$ & 104 & 1.61 & 136.5 & -1.06 & 54 \\
\hline 7. $9 \mathrm{MS} 4-11$ & $1.85^{* *}$ & 102 & $1.58 * *$ & 108 & $12.61 * *$ & 151.5 & 0.27 & 63 \\
\hline 8. $9 \mathrm{MS} 4-13$ & $3.68 * *$ & 102 & $3.24 * *$ & 107 & $20.77 * *$ & 151.5 & $17.61 * *$ & 80 \\
\hline 9. $9 \mathrm{MS} 4-15$ & 0.52 & 99 & 0.08 & 106 & $-6.23 *$ & 113 & $-6.73 * *$ & 55.5 \\
\hline 10.9MS4-16 & -0.15 & 100 & 0.24 & 106 & $11.94 * *$ & 148 & $14.44 * *$ & 69.5 \\
\hline 11.9MS4-18 & $-1.65 * *$ & 100 & -0.76 & 107 & -3.06 & 112.5 & $-4.23 * *$ & 51.5 \\
\hline $\mathrm{SE}_{(} g_{i)}$ & 0.723 & - & 0.669 & - & 5.832 & - & 3.636 & - \\
\hline $\mathrm{SE}_{\left(g_{i-} g_{j}\right)}$ & 1.252 & - & 1.158 & - & 10.101 & - & 6.298 & - \\
\hline
\end{tabular}

$* \mathrm{P}=0.05$ and $* * \mathrm{P}=0.01$ 
Table 3. contd.

\begin{tabular}{|c|c|c|c|c|c|c|c|c|}
\hline \multirow[t]{2}{*}{ Parents } & \multicolumn{2}{|c|}{ Ear length } & \multicolumn{2}{|c|}{ No. of grains/ear } & \multicolumn{2}{|c|}{ 1000-grain weight } & \multicolumn{2}{|c|}{ Yield (t/ha) } \\
\hline & gca & mean & gca & mean & gca & mean & gca & mean \\
\hline \multicolumn{9}{|l|}{ Testers: } \\
\hline 1.L 22 & -0.05 & 13 & -7.76 & 329 & 0.60 & 305 & -0.313 & 3.77 \\
\hline 2.CML 431 & 0.13 & 10.67 & -26.67 & 251 & 6.87 & 280 & -0.226 & 3.54 \\
\hline 3.CML 486 & -0.08 & 13 & 34.42 & 282 & -7.47 & 316 & 0.539 & 3.29 \\
\hline$\overline{\mathrm{SE}_{(} g_{i}}$ & 0.28 & - & 14.49 & - & 5.74 & - & 0.32 & - \\
\hline $\mathrm{SE}_{(} g_{i-} g_{j)}$ & 0.40 & - & 20.49 & - & 8.12 & - & 0.45 & - \\
\hline \multicolumn{9}{|l|}{ Lines: } \\
\hline 1. $9 \mathrm{MS} 4-1$ & 0.12 & 10.7 & $32.70 * *$ & 150 & $-19.27 * *$ & 247.5 & $1.66^{* *}$ & 4.8 \\
\hline 2. $9 \mathrm{MS} 4-2$ & -0.04 & 9.8 & $53.70 * *$ & 80 & $-33.22 * *$ & 250 & 0.60 & 3.4 \\
\hline 3. $9 \mathrm{MS} 4-4$ & -0.02 & 9.25 & $-59.64 * *$ & 201 & -6.72 & 325.5 & $-1.43 * *$ & 4.7 \\
\hline 4. $9 \mathrm{MS} 4-5$ & -0.22 & 11.8 & -23.64 & 332 & -3.14 & 263.5 & $-1.63 * *$ & 4.9 \\
\hline 5. 9MS4-7 & 0.17 & 12 & -12.97 & 216 & $37.58 * *$ & 270 & $-0.82 *$ & 6.1 \\
\hline 6. 9MS4-9 & -0.12 & 18 & -11.64 & 288 & $9.98 *$ & 301 & $-0.56^{*}$ & 3.4 \\
\hline 7. 9MS4-11 & -0.10 & 11.9 & 12.03 & 105 & $18.98^{* *}$ & 297.5 & $1.09 * *$ & 3.8 \\
\hline 8. 9MS4-13 & 0.14 & 10.7 & $32.03 *$ & 259 & -6.34 & 266.1 & 0.67 & 6.6 \\
\hline 9. 9MS4-15 & -0.04 & 11.25 & 2.36 & 232 & $-30.04 * *$ & 285 & $-0.91 *$ & 6.4 \\
\hline 10.9MS4-16 & 0.14 & 11.2 & 21.70 & 241 & $24.31 * *$ & 280 & $1.27 * *$ & 6.3 \\
\hline 11.9MS4-18 & -0.02 & 11 & $-46.64 * *$ & 196 & 7.89 & 291 & 0.06 & 5.5 \\
\hline$\overline{\left.\mathrm{SE}_{(} g_{i}\right)}$ & 0.54 & - & 27.7 & - & 11.00 & - & 0.61 & - \\
\hline $\mathrm{SE}_{\left(g_{i-} g_{j}\right)}$ & 0.951 & - & 48.06 & - & 19.059 & - & 1.06 & - \\
\hline
\end{tabular}

$* \mathrm{P}=0.05$ and $* * \mathrm{P}=0.01$.

The gca effects of parents indicated that the line $9 \mathrm{MS} 4-1,9 \mathrm{MS}_{4}-4$ and $9 \mathrm{MS}_{4-}$ 5 exhibited significant negative gca effects for tasseling and silking which could be utilized for evolving earliness. Roy et al. (1998), Hussain et al. (2003) and Uddin et al. (2006) also observed similar phenomenon in their study. For both plant height and ear height the lines $9 \mathrm{MS}_{4}-4,9 \mathrm{MS}_{4}-5$ and $9 \mathrm{MS}_{4}-7$ contributed highly significant negative effects for evolving shorter plant and ear height. This indicated that these parents could be utilized for developing early and dwarf hybrids. Only four lines $9 \mathrm{MS}_{4}-7,9 \mathrm{MS}_{4}-9,9 \mathrm{MS}_{4}-11$ and $9 \mathrm{MS}_{4}-16$ had significant positive gca effect that could be utilized for evolving bold grain. The lines $9 \mathrm{MS}_{4-}$ $1,9 \mathrm{MS}_{4}-2$ and $9 \mathrm{MS}_{4}-13$ could be used for obtaining higher grain numbers. The lines $9 \mathrm{MS}_{4}-1,9 \mathrm{MS}_{4}-11$ and $9 \mathrm{MS}_{4}-16$ expressed highly significant positive gca effects, indicated that these parents were good general combiner and could be used for exploiting more positive alleles for yield (Table 3). These lines could be 
extensively utilized for evolving high yields. Significant gca effect for yield in maize was also reported by Paul and Duara (1991) and Ivy and Hawlader (2000). As gca is generally associated with additive gene action in inheritance of characters, the lines and testers with high gca may be utilized in hybridization programme to improve a particular trait through transgressive segregation.

\section{Specific combining ability effects (sca)}

Specific combining ability and mean of the crosses for grain yield, its components and other characters are presented in Table 4. In this investigation, significant negative sca effect were estimated from the cross 9MS4-16 $\times$ L431 for plant height. In case of maize, negative value is expected for this trait to develop short stature plant. A significant positive sca effect for 1000-grain weight was observed in 9MS4-2 $\times$ L22. Positive sca effect is expected for this yield component. In case of grain yield, only 9 MS4-1 $\times$ L22 exhibited significant positive sca effects. This cross also had high mean values for grain yield. In general, crosses involving both good general combiner as well as one good and other poor combiner showed high sca effects which are due to additive $\times$ additive and additive $\times$ dominant gene action. These results were in agreement with the earlier findings of Das and Islam (1994) in maize.

The inbred parents $9 \mathrm{MS}_{4}-1,9 \mathrm{MS}_{4}-11$ and $9 \mathrm{MS}_{4}-16$ have been identified as the best general combiner due to their good combining ability effects and also their ability to transmit characters to their progenies for most of the characters. The cross 9MS4-1 × L 22, 9MS4-1 × L486, 9MS4- $2 \times$ L431, 9MS4-11 × L486 and 9MS4-15 $\times$ L431 were identified as the best combinations for yield due to their higher sca effects along with mean performance for yield traits.

Table 4. Specific combining ability (sca) effects and mean of the crosses for grain yield, its components and other characters in maize.

\begin{tabular}{|c|c|c|c|c|c|c|c|c|c|}
\hline \multirow{2}{*}{$\begin{array}{c}\text { Sl. } \\
\text { No. }\end{array}$} & \multirow{2}{*}{ Crosses } & \multicolumn{2}{|c|}{ Days to tassel } & \multicolumn{2}{|c|}{ Days to silk } & \multicolumn{2}{|c|}{ Plant height } & \multicolumn{2}{|c|}{ Ear height } \\
\hline & & sca & mean & sca & mean & sca & mean & sca & mean \\
\hline 1. & 9MS4-1 × L 22 & 0.52 & 93 & 0.68 & 99 & 0.11 & 188.5 & -0.44 & 102.5 \\
\hline 2. & 9MS4-1 $\times$ L4 & -1.08 & 92 & -1.00 & 98 & 15 & 180 & -2.44 & 93.5 \\
\hline 3. & 91 & 0.56 & 91 & & 97 & & 187 & & 98.5 \\
\hline 4. & $9 \mathrm{MS} 4-2 \times \mathrm{L} 2$ & -1 . & . & -1.65 & 99 & 1.94 & 183.5 & -4.11 & 92 \\
\hline 5. & 9MS4-2 × L431 & 1.42 & 96 & 1.17 & 102 & 0.99 & 173 & 2.89 & 92 \\
\hline 6. & $9 \mathrm{MS} 4-2 \times \mathrm{L} 48$ & 0. & 92 & 0. & 00 & -2.92 & 178.5 & 1. & 90 \\
\hline 7. & $9 \mathrm{MS} 4-4 \times$ L 22 & 0.6 & 92 & 0.6 & 99 & 3.61 & 177 & 8.89 & 90.5 \\
\hline 8. & 9MS4-4 × L431 & 0.09 & 92 & 0.00 & 99 & -0.85 & 163 & -1.61 & 73 \\
\hline
\end{tabular}




\begin{tabular}{|c|c|c|c|c|c|c|c|c|c|}
\hline \multirow{2}{*}{$\begin{array}{c}\text { Sl. } \\
\text { No. }\end{array}$} & \multirow{2}{*}{ rosses } & \multicolumn{2}{|c|}{ Days to tassel } & \multicolumn{2}{|c|}{ Days to silk } & \multicolumn{2}{|c|}{ Plant height } & \multicolumn{2}{|c|}{ Ear height } \\
\hline & & sca & mean & sca & mean & sca & mean & sca & mean \\
\hline 9. & 9 MS4-4 × L486 & -0.77 & of & -0.68 & 30 & 6 & 70.5 & -7.29 & 7 \\
\hline 10. & 9 MS4-5 × L 22 & -0.1 & 91 & -0.3 & 98 & -7.73 & 166 & -4.27 & 36.5 \\
\hline 11. & 9MS4-5 × L431 & 0. & 92 & 0.50 & 99 & 8.82 & 173 & 9.73 & 93.5 \\
\hline 12. & 9MS4-5 × L486 & -0.1 & 89 & -0.18 & 96 & -1.09 & 172.5 & -5.46 & 78 \\
\hline 13. & 9MS4-7 × L 22 & 0.18 & 94 & 0.52 & 100 & 2.44 & 165 & 2.23 & 88 \\
\hline 14. & 9MS4-7 × L431 & 1.09 & 96 & 0.8 & 101 & 2.99 & 56 & 2.23 & 81 \\
\hline 15. & 9MS4-7 × L486 & -1.27 & 91 & -1.35 & 97 & -5.42 & 157 & -4.46 & 74 \\
\hline 16. & 9 MS4-9 × L 22 & 1.68 & 97 & 1.5 & 103 & -7.56 & 177.5 & 3.06 & 97.5 \\
\hline 17. & $9 \mathrm{M}$ & -0 & 96 & -0 . & 102 & 4.49 & 80 & -2 . & 84.5 \\
\hline 18. & 9MS4-9 × L486 & -1 & 92 & -0 . & 99 & 3.08 & 106 & -0. & 87 \\
\hline 19. & 9 MS4-11 × L 22 & -2. & 93 & -1.99 & 100 & -5.56 & 190.5 & -6.77 & 89 \\
\hline 20. & 9 MS4-11 × L431 & 0.92 & 97 & 0.8 & 103 & 2.49 & 189 & -2.77 & 86 \\
\hline 21. & 9 MS4-11 × L486 & 1. & 95 & 1 & 101 & 3.08 & 19 & 9. & 98 \\
\hline 22. & 9MS4-13 × L 22 & -0 & 97 & 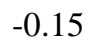 & 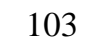 & 0.00 & 204 & -0 & 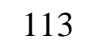 \\
\hline 23. & 9MS4-13 × L431 & -1.41 & 97 & -1 & 10 & 6. & 201 & 1. & 108 \\
\hline 24. & 9 MS4-13 × L486 & 1. & 97 & 1. & 103 & -6.09 & 198 & -1. & 104 \\
\hline 25. & 9MS4-15 × L 22 & -0.15 & 94 & 0.02 & 100 & -9.23 & 168 & -9.27 & 79.5 \\
\hline 26. & 9 MS4-15 × L431 & 0.76 & 96 & 0.8 & 102 & 1.82 & 169.5 & -1.77 & 80 \\
\hline 27. & 9 MS4-15 × L486 & -0.6 & 92 & -0.8 & 98 & 7.41 & 184.5 & 11.05 & 92.5 \\
\hline 28. & 9MS4-16 × L 22 & & 94 & -0.15 & 100 & 19.11 & 214.5 & 4.06 & 114 \\
\hline 29. & $5 \times L 431$ & -1.0 & 93 & -0 & 101 & $-23.85 * *$ & 162 & 0.06 & 103 \\
\hline 30. & 9 MS4-16 × L486 & 0.56 & 92 & 0.4 & 99 & 4.74 & 200 & -4.12 & 98.5 \\
\hline 31. & 9MS4-18 × L22 & 1.02 & 93 & 08 & 100 & 3.11 & 183.5 & 6.7 & 98 \\
\hline 32. & 9 MS4-18 × L431 & -0.58 & 92 & -0.83 & 99 & -4.35 & 166.5 & -5.27 & 79 \\
\hline 33. & 9MS4-18 × L486 & -0.44 & 90 & -0.02 & 98 & 1.24 & 181.5 & -1.45 & 82.5 \\
\hline & & 1.25 & - & 11 & - & 10.10 & - & 6.29 & - \\
\hline & $\mathrm{SE}_{(S i j-S}$ & 1.77 & - & 1.63 & - & 14.28 & - & 8.90 & - \\
\hline
\end{tabular}

$* \mathrm{P}=0.05$ and $* * \mathrm{P}=0.01$ 
Table 4. cont'd.

\begin{tabular}{|c|c|c|c|c|c|c|c|c|c|}
\hline \multirow{2}{*}{$\begin{array}{l}\text { S1. } \\
\text { No. }\end{array}$} & \multirow[t]{2}{*}{ Crosses } & \multicolumn{2}{|c|}{ Ear length } & \multicolumn{2}{|c|}{ Grains/ear } & \multicolumn{2}{|c|}{$\begin{array}{c}\text { 1000-grain } \\
\text { weight }\end{array}$} & \multicolumn{2}{|c|}{ Yield (t/ha) } \\
\hline & & sca & mean & sca & mean & sca & mean & sca & mean \\
\hline 1. & 9MS4-1 × L 22 & -0.32 & 13.9 & 7.76 & 392 & -12.37 & 273 & $3.02 * *$ & 11 \\
\hline 2. & 9MS4-1 × L431 & 0.24 & 11.8 & -77.33 & 288 & -11.13 & 280 & $-3.40 * *$ & 4 \\
\hline 3. & 9MS4-1 × L486 & 0.08 & 15.9 & 69.58 & 496 & 23.50 & 300 & 0.38 & 9 \\
\hline 4. & 9 MS4- $2 \times$ L 22 & 0.27 & 13.5 & -6.24 & 399 & $62.38 * *$ & 333 & -0.35 & 6 \\
\hline 5. & 9MS4-2 × L431 & -0.16 & 14.3 & 12.67 & 399 & $-41.73 * *$ & 235 & 1.52 & 8 \\
\hline 6. & $9 \mathrm{MS} 4-2 \times \mathrm{L} 486$ & -0.11 & 14.8 & -6.42 & 441 & -20.65 & 242 & -1.17 & 6 \\
\hline 7. & 9MS4-4 × L 22 & -0.17 & 11.1 & 2.09 & 294 & 1.43 & 299 & -0.39 & 4 \\
\hline 8. & 9MS4-4 × L431 & -0.22 & 9.7 & -28.00 & 245 & 5.92 & 310 & -0.70 & 4 \\
\hline 9. & 9MS4-4 × L486 & 0.38 & 13.8 & 25.91 & 360 & -7.35 & 282 & 1.09 & 6 \\
\hline 10. & 9 MS4-5 × L 22 & 0.10 & 12.1 & 8.09 & 336 & -9.60 & 291 & -0.33 & 4 \\
\hline 11. & 9MS4-5 × L431 & 0.05 & 13.0 & -9.00 & 300 & 2.24 & 310 & 0.52 & 5 \\
\hline 12. & $9 \mathrm{MS} 4-5 \times \mathrm{L} 486$ & -0.15 & 13.3 & 0.91 & 371 & 7.37 & 300 & -0.19 & 5 \\
\hline 13. & 9MS4-7 × L 22 & -0.10 & 14 & 4.42 & 343 & 33.38 & 375 & -1.21 & 4 \\
\hline 14. & 9MS4-7 × L431 & -0.09 & 15 & -1.67 & 318 & -15.23 & 333 & 0.07 & 5 \\
\hline 15. & 9MS4-7 × L486 & 0.19 & 14.3 & -2.76 & 378 & -18.15 & 316 & 1.14 & 7 \\
\hline 16. & 9MS4-9 × L 22 & -0.14 & 13.9 & 58.09 & 398 & -1.77 & 312 & 0.33 & 6 \\
\hline 17. & 9MS4-9 × L431 & 0.07 & 12 & -20.00 & 301 & -3.58 & 317 & -0.09 & 5 \\
\hline 18. & 9MS4-9 × L486 & 0.06 & 12.4 & -38.09 & 344 & 5.35 & 311 & -0.24 & 6 \\
\hline 19. & $9 \mathrm{MS} 4-11 \times \mathrm{L} 22$ & 0.10 & 13 & -21.58 & 342 & -19.82 & 303 & -1.83 & 5 \\
\hline 20. & $9 \mathrm{MS} 4-11 \times \mathrm{L} 431$ & 0.18 & 13.2 & 7.33 & 352 & 26.12 & 356 & 0.56 & 8 \\
\hline 21. & 9MS4-11 × L486 & -0.28 & 14.7 & 14.24 & 420 & -6.30 & 309 & 1.27 & 9 \\
\hline 22. & 9 MS4-13 × L 22 & 0.02 & 13 & 8.42 & 392 & -26.70 & 271 & -0.44 & 6 \\
\hline 23. & 9MS4-13 × L431 & 0.01 & 13.2 & -14.67 & 350 & 21.44 & 326 & -0.13 & 7 \\
\hline 24. & 9MS4-13 × L486 & -0.03 & 13.8 & 6.24 & 432 & 5.27 & 295 & 0.57 & 8 \\
\hline 25. & $9 \mathrm{MS} 4-15 \times \mathrm{L} 22$ & 0.21 & 11 & -60.91 & 293 & 23.50 & 298 & -0.12 & 5 \\
\hline 26. & 9MS4-15 × L431 & 0.06 & 14 & 89.00 & 424 & -6.67 & 274 & 2.07 & 8 \\
\hline 27. & 9MS4-15 × L486 & -0.27 & 11.9 & -28.09 & 368 & -16.83 & 249 & -1.96 & 4 \\
\hline 28. & 9 MS4-16 × L 22 & -0.23 & 14.1 & 3.76 & 377 & $-47.90 * *$ & 281 & 0.55 & 8 \\
\hline 29. & 9MS4-16 × L431 & -0.06 & 13.7 & 12.67 & 367 & 26.29 & 361 & -0.69 & 7 \\
\hline 30. & 9MS4-16 × L486 & 0.29 & 14.5 & -16.42 & 399 & 21.62 & 342 & 0.15 & 8 \\
\hline 31. & 9MS4-18 × L22 & 0.26 & 15.5 & -3.91 & 301 & -2.54 & 310 & 0.76 & 7 \\
\hline 32. & 9MS4-18 × L431 & -0.09 & 12.4 & 29.00 & 315 & -3.65 & 315 & 0.27 & 6 \\
\hline \multirow[t]{3}{*}{33.} & 9MS4-18 × L486 & -0.17 & 12.4 & -25.09 & 322 & 6.18 & 310 & -1.03 & 6 \\
\hline & $\mathrm{SE}_{\left(s_{i j}\right)}$ & 0.95 & - & 48.06 & - & 19.05 & - & 1.06 & - \\
\hline & $\mathrm{SE}_{\left({ }_{i j-}\right.} s_{k l)}$ & 1.34 & - & 67.97 & - & 26.95 & - & 1.49 & - \\
\hline
\end{tabular}

$* \mathrm{P}=0.05$ and $* * \mathrm{P}=0.01$ 


\section{Conclusion}

The good combining crosses $9 \mathrm{MS}_{4}-1 \times \mathrm{L}_{2} 2,9 \mathrm{MS}_{4}-1 \times \mathrm{L}_{4} 86,9 \mathrm{MS}_{4}-2 \times \mathrm{L}_{4} 31$, $9 \mathrm{MS}_{4}-11 \times \mathrm{L} 486$ and $9 \mathrm{MS}_{4}-15 \times \mathrm{L} 431$ could be used in future breeding program to develop high yielding hybrids with desirable traits.

\section{References}

Das, U.R. and M.H. Islam. 1994. Combining ability and genetic studies for grain yield and its components in maize (Zea mays L.). Bangladesh J. Pl. Breed. Genet. $7(2): 41-47$

Hussain, S. A., M. Amiruzzaman and Z. Hossain. 2003. Combining ability estimates in maize. Bangladesh J. Agril. Res. 28(3):435-440.

Ivy, N.A. and M.S. Howlader. 2000. Combining ability in maize. Bangladesh J. Agril. Res. 25: 385-392.

Kempthorne, O. 1957. An Introduction to Genetic Statistics. New York: John Wiley \& Sons, Inc. London: Chapman \& Hall Ltd. pp. 458-471.

Mahto, R.N. and D.K. Ganguly, , 2001, Heterosis and combining ability studies in maize (Zea mays L.). J. Res. Brirsa Agric. Univ. 13: 197-199.

Narro, L., S. Pandey, J. Crossa, C. D. Leon, and F. Salazar. 2003. Using line $\times$ tester interaction for the formation of yellow maize synthetics tolerance to acid soils. Crop Sci. 43: $1717-1728$.

Parvin, S. 2009. Annual Research Report, Plant Breeding Division, BARI, Pp: 11-18.

Paul, S.K. and R.K. Duara.1991. Combining ability studies in maize (Zea mays L.). Intl. J. Tropics. Agric. 9(4):250-254.

Roy, N. C., S.U. Ahmed. A. S. Hussain and M. M. Hoque. 1998. Heterosis and combining ability analysis in maize (Zea mays L.). Bangladesh J. Pl. Breed. Genet.11(1\&2):35-41.

Singh, D. N. and Singh, I. S., 1998, Line $\times$ tester analysis in maize (Zea mays L.). J. Res. Agric. Univ. 10: 177-182.

Singh, R.K. and B.D. Chaudhary. 1979. Biometrical methods in quantitative genetic analysis. Kalyani Publs, New Delhi, Ludhiana, India. Pp: 127-223.

Sofi, P. and A. G. Rather. 2006. Genetic analysis of yield trails in local and CIMMYT inbred line crosses using Line $\times$ tester analysis in maize (Zea mays L.). Asian $J$. Plant Sci. 5 (6): 1039-1042.

Suneetha, Y., Patel, J. R. and Srinivas, T., 2000, Studies on combining ability for forage characters in maize (Zea mays L.). Crop Res. 9: 226-270.

Uddin, M. S., F. Khatun, S. Ahmed, M. R. Ali and S. A. Bagum. 2006. Heterosis and Combining Ability in Field Corn (Zea mays L.). Bangladesh J. Bot. 35 (2): 109-116. 